Revue

Revue de l'histoire des religions

de Ihistoire des religions

$3 \mid 2019$

Varia

\title{
Les visions dans le livre d'Ézéchiel
}

Notes critiques

Visions in the Book of Ezekiel. Critical notes

Stéphanie Anthonioz

\section{(2) OpenEdition}

Journals

Édition électronique

URL : https://journals.openedition.org/rhr/9913

DOI : 10.4000/rhr.9913

ISSN : 2105-2573

Éditeur

Armand Colin

Édition imprimée

Date de publication : 1 septembre 2019

Pagination : $577-589$

ISBN : 978-2-200-93260-2

ISSN : 0035-1423

Référence électronique

Stéphanie Anthonioz, "Les visions dans le livre d'Ézéchiel », Revue de l'histoire des religions [En ligne],

3 | 2019, mis en ligne le 01 janvier 2022, consulté le 08 janvier 2022. URL : http://

journals.openedition.org/rhr/9913; DOI : https://doi.org/10.4000/rhr.9913

Tous droits réservés 


\section{Les visions dans le livre d'Ézéchiel Notes critiques*}

Cette contribution est une recension critique de l'ouvrage de Janina Maria Hiebel, Ezekiel's Vision Accounts as Interrelated Narratives: A Redaction-Critical and Theological Study, qui étudie selon une approche rédactionnelle et théologique la vision continuée du livre d'Ézéchiel (1,1-3,15;3,22-27 ; 8,1-11,25;37,1-14;40,1-43,12 ;44,1-2.4-6 ;47,1-12) et conclut que la vision est le fruit de réécritures successives. S'il ne fait pas de doute que la vision comme l'ensemble du livre d'Ézéchiel d'ailleurs n'est pas l'œuvre d'une seule main de scribe, il est permis cependant de questionner le découpage systématique du texte lorsque l'on considère sa cohérence d'ensemble. Cette note poursuit un débat méthodologique sur le bien-fondé d'une pratique dite historique qui pourtant sépare textes et contextes.

\section{Visions in the Book of Ezekiel. Critical notes.}

This contribution is a critical review of Janina Maria Hiebel's Ezekiel's Vision Accounts as Interrelated Narratives : A Redaction-Critical and Theological Study, that studies the continued vision in the book of Ezekiel (1:1-3:15; 3:22-27; 8:1-11:25; 37:1-14; 40:1-43:12 ; 44:1-2.4-6; 47:1-12) according to a redactional and theological approach and concludes that it is the fruit of successive rewritings. While there is no doubt that the vision, like the book itself, is not the work of a single scribe, one may however question the systematic subdivisions of the text in light of its coherence. This note seeks to pursue a methodological debate concerning the legitimacy of a practice that is called historical even though it separates texts and contexts.

* À propos de Janina Maria Hiebel, Ezekiel's Vision Accounts as Interrelated Narratives: A Redaction-Critical and Theological Study, Berlin, de Gruyter ("Beihefte zur Zeitschrift für die alttestamentliche Wissenschaft", 475), 2015, 393 p., 119,95€ / \$168.00 / £108.99. ISBN 978-3-11-040665-8. 
L'ouvrage publié dans la célèbre collection BZAW est une version remaniée de la thèse doctorale soutenue par Janina Maria Hiebel à Murdoch University, en Australie, l'année 2014. Autour de cette publication, deux articles ont été produits, «Redaction, Rhetoric and a New Beginning in Ezekiel 1,1-3,15», Australian Biblical Review, 64, 2016 p. 14-24 et «Visions of Death and Re-Creation: Ezekiel 8-11, 37:1-14 and the Crisis of Identity in the Babylonian Exile and Beyond», Pacifica, 28, 2015, p. 243-255. Le projet de l'auteure est de revisiter les visions du livre d'Ézéchiel sous l'angle historico-critique et théologique. Après la table des matières (p. v-xiii), les remerciements (p. xvii) et la liste des abréviations (p. xix-xxii), l'introduction se présente de manière claire et concise (p.1-49) et permet de situer le cadre dans lequel l'étude va être déployée.

Tout d'abord, Ézéchiel est considéré comme le premier auteur de son livre. Certes l'ensemble et les visions, en particulier, ont été relues et donc inscrites dans une histoire rédactionnelle, il n'en reste pas moins que l'auteur du rouleau porte le nom prophétique d'Ézéchiel et répond à la définition sacerdotale et d'élite déportée qui est faite de lui. Ensuite, l'exercice du status quaestionis est conduit aussi brièvement que remarquablement (1.2 Literature Review, p. 4-35). Il se divise en deux parties. La première concerne les approches générales du livre selon un modèle triple : - l'école ou groupe de disciples actifs surtout pendant l'exil (1.2.1.1 The «Ezekiel School» ou Fortschreibungsmodell); des rédactions conflictuelles qui s'étendent sur la durée longue (1.2.1.2 Conflicting Redactions); - le modèle holistique ou synchronique qui s'intéresse à l'unité littéraire indépendamment des questions de critique rédactionnelle (1.2.1.3 Holistic Interpretation).

La deuxième partie du status quaestionis se focalise sur la littérature des visions en prenant chacune des péricopes de manière isolée (1.2.2 Literature on Ezekiel 1:1-3:15; 1.2.3 Literature on Ezekiel 8-11; 1.2.4 Literature on Ezekiel 37:1-14; 1.2.5 Literature on Ezekiel 40-48). C'est effectivement la meilleure manière de souligner qu'aucune étude récente ne s'est intéressée 
aux visions du livre dans une approche diachronique. La dernière en date remonte à Eberhard Baumann, «Die Hauptvisionen Hesekiels : in ihrem zeitlichen und sachlichen Zusammenhang untersucht », Zeitschrift für die alttestamentliche Wissenschaft, 67, 1955, p. 56-67. Aussi le projet est-il amplement justifié: repenser les liens entre les différentes visions du livre et surtout tirer des conclusions théologiques de l'approche historico-critique (p. 36-37). L'auteure présente ensuite les critères pour définir les textes des visions et dans la lignée (avec quelques modifications) de Achim Behrens, Prophetische Visionsschilderungen im Alten Testament: Sprachliche Eigenarten, Funktion und Geschichte einer Gattung, Münster : UGARIT-Verlag (Alter Orient und Altes Testament - AOAT 292), 2002, qui identifie un double paradigme des visions associant vision et parole ou mission, retient les sections $1,1-3,15 ; 3,22-27 ; 8,1-11,25 ; 37,1-14 ; 40,1-43,12$; 44,1-2.4-6 ; 47,1-12. La méthode est ensuite exposée, l'histoire ou la critique rédactionnelle justifiée par les contradictions logiques, les changements soudains de style ou de langue (grammaire/ vocabulaire), les termes exprimant selon le contexte des concepts différents, les interruptions de la narration ou du discours, les déséquilibres de structure. Au terme de cette introduction, un plan de l'ouvrage est résumé (p. 48-49). Certainement l'introduction situe l'étude dans une démarche scientifique claire, mais peut-être aussi datée, j'y reviendrai.

La lecture se poursuit et l'analyse se révèle extrêmement chirurgicale. Chaque texte est analysé d'abord de manière indépendante, découpé dans ses moindres tensions avant d'être repensé à la lumière des autres visions. En quelque sorte, ce ne sont pas des récits isolés qui sont analysés mais une grande vision qui est reprise par étapes tout au long du livre. Pour arriver à ce résultat, le travail est laborieux. Le découpage textuel rend alors la cohérence d'ensemble difficile à saisir. D'ailleurs, la table des matières qui s'étend sur 13 pages visualise facilement ce point. Quoi qu'il en soit, quels sont les résultats obtenus?

Les résultats des textes pris indépendamment sont les suivants :

- En Ez 1,1-3,15, le lien entre la vision de la gloire en ouverture et la vision qui la suit immédiatement, vision qui est aussi l'appel et la mission du prophète, ne serait pas originel. 
L'appel prophétique doit être localisé dans les versets 2,3-3,11*, $14 \mathrm{c}, 15^{*}$ qui présentent une composition de forme concentrique suivie d'une conclusion brève. La vision de la gloire aux versets 1,1, 3b-13, 22-28; 2,1-2 ; 3,12, 14abd permet d'introduire l'appel prophétique et de le re-contextualiser. La séquence concernant les roues $(1,15-21)$ doit être considérée comme un ajout ultérieur tandis que la vision en 3,22-27 apparaît comme une compilation d'éléments présents en 2,3-3,15*.

- En Ez 8-11, le récit de la première vision du temple serait le développement de trois textes indépendants et pas moins quatre étapes rédactionnelles. Le premier récit est celui d'un jugement prophétique $\left(8,1,3\right.$ cde, $5-7 \mathrm{a}, 9-18 ; 9,1-2^{*}, 5-10^{*} ; 10,2,4,6-7^{*}$, $18 \mathrm{a}, 19 \mathrm{~d} ; 11,23-25)$. Deux passages $\left(11,3-4,7-12\right.$ et $\left.11,14-20^{*}\right)$ auraient été insérés dans ce cadre afin de lui apporter une touche d'espoir. Une révision intégrant les versets $9,2 \mathrm{e}, 3 \mathrm{~cd}, 4,5 \mathrm{~b}, 6 \mathrm{~b}_{1} \mathrm{cb}_{2}$, 11 aurait permis d'adoucir encore le jugement en excluant les innocents du carnage à venir. Les autres marques rédactionnelles impliquant le chapitre 10, si elles compliquent la structure générale, n'en créent pas moins des liens avec la vision de la gloire en Ez 1 et harmonisent donc l'ensemble.

- En Ez 37,1-14, dans la vision des ossements, le récit originel aurait inclus les versets 37,1-6, 7c-8d, 10c-12, 14a-f tout en présentant une structure promesse-accomplissement-promesse. Le processus de résurrection avec l'esprit médiateur de vie aurait été intégré en deux étapes $(37,7 \mathrm{ab}, 8 \mathrm{e}-10 \mathrm{~b}$, puis $37,13,14 \mathrm{~g})$.

- L'analyse de Ez 40-48 se limite aux sections 40,1-43,12; 44,1-2, 4-6; 47,1-12 et le récit originel à 40,1-2a*, 3b-13, 15-37, $47-49 ; 41,1-4 ; 42,15,20 b-e$; 43,1-2, 4-10. Le récit est celui d'un temple parfaitement symétrique qui semble relever plus du message théologique que du projet de construction. Puis la gloire revient depuis l'Est faisant écho à son départ en 10,19 et 11,23. La fermeture des portes orientales $(44,1-2)$ comme la vision de la rivière $(47,1-10,12)$ seraient, parmi les développements de l'époque exilique tardive, un moyen de décrire les bienfaits de la présence divine retrouvée.

Ces résultats ne sont pas totalement nouveaux, comme le concède l'auteure, et rejoignent souvent ceux d'autres exégètes. Mais la nouveauté réside dans la manière de penser la diachronie sur l'ensemble de ces textes et de présenter une histoire commune 
de leur rédaction. Ainsi, les visions originelles $\left(2,3-3,15^{*} ; 8-11^{*}\right.$; $\left.37,1-14^{*} ; 40,1-43,10+11,3-12^{*}, 14-20^{*}\right)$ sont attribuées à l'auteur-narrateur-prophète Ézéchiel du début de l'exil. Ces quatre visions originelles mettent en valeur l'intérêt pour le sanctuaire comme pour le prophète et opposent jugement et restauration. Peu après, dans un moment de perte de confiance, la vision de la gloire aurait été introduite $\left(1,1-2,2^{*} ; 3,12-14^{*}\right)$ afin d'offrir un regain de confiance tout en confirmant l'annonce prophétique. Les liens entre les différentes visions se développent alors comme, par exemple la rédaction concernant les Chérubins $(1,5-26 ; 10)$. Ainsi, l'ajout des roues en 1,15-21 aurait été recopié en 10,9-17*. De nouveaux ajouts dans le style de cette grande vision unifiée auraient ensuite vu le jour à une époque plus tardive $\left(3,22-27 ; 44,4-6^{*}\right)$, sans oublier les références anaphoriques qui renvoient d'une vision à l'autre $(3,23 ; 8,4 ; 10,13,15,20,22 ; 43,3)$.

La deuxième partie qui est supposée renouveler la lecture théologique du livre apparaît plus maigre (p. 259-317). L'idée est de prendre en compte le développement historique pour enrichir la dimension théologique. En réalité, les résultats sont décevants. Dans les visions originelles, si le récit passe par un «je » prophétique, il n'en reste pas moins que le personnage essentiel des visions est la divinité Yhwh. Le point de vue est toujours divin. Les relectures développent manifestement la puissance et la gloire divines par opposition à un prophète de plus en plus passif (3,22-27). Le développement anthropologique introduit le concept de "grâce » et un développement sur les créatures hybrides amène à les considérer comme des symboles sans personnalité propre qui ne font que servir certains aspects divins. Ils deviennent alors symptomatiques d'une représentation divine de plus en plus transcendante. Quatre appendices suivent et présentent le texte hébreu (avec un apparat critique succinct) et la traduction de l'auteure (p. 326-366), une bibliographie (p. 367-378), enfin l'index des références scripturaires (p. 379-394).

Le sentiment du présent recenseur est mitigé. Si la méthode historico-critique n'a pas nécessairement besoin de redorer ses blasons, elle n'est pas sans poser des problèmes évidents. C'est peut-être précisément pour cette raison que l'auteure a tenté une deuxième partie théologique, mais qui s'avère sans 
résultat important dans l'ordre de la recherche. Quels sont les «problèmes » méthodologiques ? Je les résumerai par quelques questions : comment peut-on, avec l'avancée des approches littéraires et historiques justement, continuer à penser un prophète auteur-narrateur sans distinguer la mise-en-scène et la fiction inhérente à toute ouvre de littérature ou de pensée ? Comment circonscrire cet auteur et ses relecteurs ultérieurs à son texte seul comme s'il(s) vivai(en)t (dans un cachot) sans autre interaction, sans autre contact, bref sans autre lecture que le livre (son livre), de sorte que Ézéchiel se réécrit en Ézéchiel ? Certes les textes bibliques sont pour le moins contradictoires mais peut-on sans autre lumière faire sens de leurs contradictions internes?

Ces questions invitent à nous situer plutôt dans la fiction savamment construite. Comme l'a souligné M. Nissinen, il faut définitivement distinguer prophètes et livres prophétiques :

The figure of the prophet Ezekiel is "a first-person sympathetic narrator," through whom the reader is supposed to experience "all speech, all action." In other words, "[t]he real Ezekiel is the symbolic Ezekiel, who is more than the historical Ezekiel." The figure of the prophet is already part of the literary construct of the book, which (rather than the historical framework) is the primary context of his identity. ${ }^{1}$

La même remarque vaut pour le cadre historique de l'exil lorsque les exégètes comme J. M. Hiebel suivent scrupuleusement le système de datation interne au livre (593-571 av. n. è.).

Pour sortir de cette impasse, il paraît impossible de traiter des visions dans le livre d'Ézéchiel sans prendre en compte le corpus plus large des visions divines, d'une part et, d'autre part, l'apport des sources iconographiques et des travaux comparatistes. Ces sources permettraient non seulement d'inscrire le livre dans l'histoire des idées, celle de la vision comme de la représentation divine, mais également de saisir les influences au cœur de la rédaction, des influences propres à la bibliothèque de Jérusalem (comme par exemple le livre d'Isaïe), ou des influences plus internationales et peut-être spécifiquement babyloniennes. D'ailleurs, cette critique peut s'appliquer au sujet de la délimitation

1. Martti Nissinen, «(How) Does the Book of Ezekiel Reveal Its Babylonian Context?», Die Welt des Orients, 45, 2015, p. 87. 
des passages retenus. Il ne fait pas de doute que l'analyse serait impossible si des textes n'étaient pas délimités. Cependant, leur interprétation ne peut se faire sans un rapport étroit avec le contexte littéraire dans lequel ils sont insérés : les couper de ce contexte me semble fausser leur interprétation, comme je voudrais le montrer.

Prenons, à titre d'exemple, l'identification des Chérubins (Ez 10) avec les «Vivants » (Ez 1). La vision sans aucun doute est continuée et les Chérubins, familiers des sources bibliques, même si leur étymologie n'est pas assurée ${ }^{2}$, sont décrits selon le modèle des «Vivants ». Les roues reçoivent le nom de «cercle » ou 《tourbillon» au singulier (גלגל, (10,2.6.13), à comprendre sans doute comme leur agencement dans le mouvement. Le thème de la purification développe clairement Is 6,6-7, à ceci près que ce n'est pas le prophète qui doit être purifié mais la ville Jérusalem $(10,2)^{3}$. Une plénitude remplit alors le temple mais il s'agit de «nuée » (ענן, עשן) non de «fumée Is $6,4)$, ce qui n'est pas sans ancrer cette vision dans celle du Sinaï (« dans un nuage de nuée », Ex 19,9; «épaisse nuée », 19,16 et encore 24,$18 ; 33,9-10)$. Cette plénitude enfumée s'accompagne d'une dimension sonore: on peut ainsi comparer le bruit des ailes et la voix du tout-puissant (10,5 cf Is 6,3). La description des Chérubins, tout en s'inscrivant dans les traditions bibliques de théophanie, reprend donc celle des Vivants, dans un ordre pratiquement chronologique avec quelques exceptions notoires expliquées ci-après.

Vivants et Chérubins $(\mathrm{Ez} 1 ; 10)^{4}$

2. Pour Alice Wood, Of Wings and Wheels: A Synthetic Study of the Biblical Cherubim, Berlin, de Gruyter (BZAW 385), 2008, un lien possible avec la racine akkadienne kurību ne peut constituer un argument en faveur d'un rôle d'intercesseur des Chérubins. Contra Édouard Dhorme, «Les Chérubins », Revue biblique, 35, 1926, p. 328-358 et 481-495.

3. Comparer la gestuelle du Séraphin et celle du Chérubin (Is 6,6,7 ; Ez 10,7) avec les actions d'étendre la main, de prendre et de mettre/toucher.

4. La traduction qui est proposée du texte massorétique est une traduction littérale, elle laisse de manière apparente les contradictions nombreuses de nombre et surtout de genre. Car les Vivants / Chérubins sont tantôt traités comme des masculins grammaticalement, tantôt comme des féminins. 


\begin{tabular}{|c|c|}
\hline Ez 1 1 Les Vivants» (החיות) & Ez 10 « Les Chérubins » (הכרובים) \\
\hline $\begin{array}{l}\text { 5et, du milieu (du feu), la ressem- } \\
\text { blance de quatre (créatures) vivantes; } \\
\text { et voici leur aspect : la ressemblance } \\
\text { d'un homme elles avaient; } \\
{ }^{6} \text { et quatre faces chacune, et quatre } \\
\text { ailes chacune ils avaient; } \\
{ }^{7} \text { et leurs jambes / pieds étaient une } \\
\text { jambe/un pied droit(e) ; et la plante } \\
\text { de leurs pieds était comme la plante } \\
\text { d'un pied de veau ; et ils étince- } \\
\text { laient comme l'apparence de l'ai- } \\
\text { rain poli ; } \\
{ }^{8} \text { et il y avait des mains d'homme } \\
\text { au-dessous de leurs ailes sur leurs } \\
\text { quatre côtés; leurs faces et leurs } \\
\text { ailes, ils avaient (tous) les quatre. } \\
{ }^{9} \text { Étaient jointes l'une à l'autre } \\
\text { leurs ailes ; ils ne se tournaient pas } \\
\text { lorsqu'elles allaient : chacun dans la } \\
\text { direction de sa face / droit devant soi, } \\
\text { ils allaient. (...) }\end{array}$ & $\begin{array}{l}{ }^{8} \mathrm{Et} \text { on voyait aux Chérubins } \\
\text { le modèle d'une main d'homme } \\
\text { sous leurs ailes (cf } 8,3) \text {. }\end{array}$ \\
\hline $\begin{array}{l}{ }^{15} \mathbf{E t} \text { je regardais les (créatures) } \\
\text { vivantes, et voici, une roue sur la } \\
\text { terre, à côté des (créatures) vivantes, } \\
\text { vers leurs quatre faces. } \\
{ }^{16} \mathbf{L} \text { 'aspect des roues et leurs struc- } \\
\text { tures étaient comme l'apparence } \\
\text { d'un chrysolithe, et il y avait une } \\
\text { même ressemblance pour les } \\
\text { quatre (créatures). Et quant à } \\
\text { leur aspect et leur structure, c'était } \\
\text { comme si une roue était au milieu } \\
\text { d'une roue. }\end{array}$ & $\begin{array}{l}\text { et l'aspect des roues était comme } \\
\text { l'apparence d'une pierre de chryso- } \\
\text { lithe. } \\
{ }^{10} \text { Et quant à leur aspect, il y avait } \\
\text { une même ressemblance pour les } \\
\text { quatre, comme si une roue était au } \\
\text { milieu d'une roue. }\end{array}$ \\
\hline $\begin{array}{l}{ }^{17} \text { Vers leurs quatre côtés, en allant, } \\
\text { ils allaient, ils ne se tournaient } \\
\text { point quand elles allaient. }\end{array}$ & $\begin{array}{l}{ }^{11} \text { Quand ils allaient, sur leurs } \\
\text { quatre côtés ils allaient, ils ne se } \\
\text { tournaient pas, quand ils allaient } \\
\text { mais, vers le lieu où la tête se tour- } \\
\text { nait, ils allaient à leur suite : ils ne se } \\
\text { tournaient pas quand ils allaient. }\end{array}$ \\
\hline
\end{tabular}




\begin{tabular}{|c|c|}
\hline $\begin{array}{l}{ }^{18} \text { Et quant à leurs jantes / dos, } \\
\text { hauteur à eux et terreur à eux, et leurs } \\
\text { (masc.) jantes / dos étaient pleines } \\
\text { d'yeux / étincelles tout autour à } \\
\text { (toutes) les quatre. }\end{array}$ & $\begin{array}{l}{ }^{12} \text { Et tout leur corps, et leur dos, } \\
\text { et leurs mains, et leurs ailes, et les } \\
\text { roues, étaient pleins d'yeux / étin- } \\
\text { celles tout autour, à (tous) les } \\
\text { quatre, leurs roues. }\end{array}$ \\
\hline & $\begin{array}{l}{ }^{13} \text { Quant aux roues, on leur donna le } \\
\text { nom de cercle, dans mes oreilles. }\end{array}$ \\
\hline $\begin{array}{l}{ }^{10} \text { Et la ressemblance de leurs faces } \\
\text { était la face d'un homme ; et la face } \\
\text { d'un lion, sur la droite, ils avaient } \\
\text { (tous) les quatre; et la face d'un } \\
\text { bovin, sur la gauche, elles avaient } \\
\text { (toutes) les quatre; et la face d'un } \\
\text { aigle, elles avaient (toutes) les quatre. }\end{array}$ & $\begin{array}{l}{ }^{14} E t \text { chacun avait quatre faces: la } \\
\text { face du premier était la face d'un } \\
\text { Chérubin, la face du second était } \\
\text { la face d'un homme, et le troi- } \\
\text { sième était la face d'un lion, et le } \\
\text { quatrième, la face d'un aigle. }\end{array}$ \\
\hline $\begin{array}{l}{ }^{19} \text { Et quand allaient les (créatures) } \\
\text { vivantes, les roues allaient à côté } \\
\text { d'eux, et quand s'élevaient les (créa- } \\
\text { tures) vivantes de dessus la terre, } \\
\text { s'élevaient les roues. }\end{array}$ & $\begin{array}{l}{ }^{15} \text { Et les Chérubins montèrent. C'était } \\
\text { là la (créature) vivante que j'avais vu } \\
\text { près du fleuve Kevar. }\end{array}$ \\
\hline $\begin{array}{l}{ }^{20} \text { Là où le souffle devait aller, ils } \\
\text { allaient, là le souffle tendait à aller, } \\
\text { et les roues s'élevaient auprès d'eux, } \\
\text { car le souffle de la (créature) vivante } \\
\text { est dans les roues. } \\
\\
{ }^{21} \text { Quand ils allaient, ils allaient, et } \\
\text { quand ils s'arrêtaient, ils s'arrêtaient, } \\
\text { et quand ils s'élevaient de dessus la } \\
\text { terre, les roues s'élevaient auprès } \\
\text { d'eux, car le souffle de la (créature) } \\
\text { vivante est dans les roues. (...) }\end{array}$ & $\begin{array}{l}{ }^{16} \mathrm{Et} \text { quand les Chérubins allaient, les } \\
\text { roues allaient à côté d'eux, et quand } \\
\text { les Chérubins élevaient leurs ailes } \\
\text { pour monter de la terre, les roues } \\
\text { aussi ne se détournaient point d'à } \\
\text { côté d'eux. } \\
{ }^{17} \text { Quand ils s'arrêtaient, ils s'arrê- } \\
\text { taient, et quand ils montaient, ils } \\
\text { montaient avec eux, car le souffle de } \\
\text { la (créature) vivante était en elles. }\end{array}$ \\
\hline $\begin{array}{l}{ }^{28} \text { Comme l'aspect de l'arc qui est } \\
\text { dans la nuée au jour de pluie, tel était } \\
\text { l'aspect de la splendeur (tout) autour. } \\
\text { C'était là l'aspect de la ressemblance } \\
\text { de la gloire de Yhwh. Et je vis, et je } \\
\text { tombai sur ma face et j'entendis une } \\
\text { voix qui parlait. }\end{array}$ & $\begin{array}{l}{ }^{18} \text { Et la gloire de Yhwh sortit du seuil } \\
\text { de la maison, et se tint au-dessus des } \\
\text { Chérubins. }{ }^{19} \mathrm{Et} \text { les Chérubins éle- } \\
\text { vèrent leurs ailes et montèrent de } \\
\text { la terre sous mes yeux, alors qu'ils } \\
\text { sortaient, et les roues étaient auprès } \\
\text { d'eux. Et ils s'arrêtèrent à l'entrée } \\
\text { de la porte orientale de la maison de } \\
\text { Yhwh, et la gloire du Dieu d'Israël } \\
\text { était sur eux, en haut. }\end{array}$ \\
\hline
\end{tabular}




\begin{tabular}{|l|l|}
\hline Cf 1,6-8 & ${ }^{20}$ C'était là la (créature) vivante que \\
j'avais vu au-dessous du Dieu d'Is- \\
raël, près du fleuve Kevar, et je sus \\
qu'ils étaient des Chérubins. \\
${ }^{21}$ Chacun avait quatre faces, et \\
chacun, quatre ailes, et il y avait une \\
ressemblance de mains d'homme \\
sous leurs ailes. \\
\\
${ }^{22}$ Et quant à la ressemblance de leurs \\
faces, c'étaient les faces que j'avais \\
vues près du fleuve Kevar, leur \\
aspect, et eux-mêmes. Chacun dans \\
la direction de sa face, ils allaient.
\end{tabular}

Un certain nombre de modifications doivent être expliquées, car si le fil de la description semble le même, un déplacement majeur se produit concernant l'identification des quatre faces $(1,10 / / 10,14)$. La face de bovin disparaît au profit de celle d'un «Chérubin ». Cela indique-t-il l'assimilation de l'un à l'autre ? Par ailleurs, la description générale des «Vivants » est présentée en introduction $(1,5-8)$ tandis que celle des «Chérubins » se fait en conclusion $(10,20-22)$. On pourrait identifier là un phénomène de grande inclusion qui signerait l'ajout de la péricope concernant les Chérubins, expliquant par là le fait qu'ils ne sont pas mentionnés dans la vision inaugurale. De fait, un certain nombre d'études depuis J. H. Tigay ont recensé les phénomènes de réécriture 5 . Or ces mêmes conclusions pourraient s'appliquer à l'évolution de la description entre Ez 1 et $10^{6}$. En effet, on retrouve les pratiques décrites dans le passage de l'un à l'autre texte :

- développement ou plus précisément répétition qui permet d'insister sur l'aspect distributif (« une roue à côté d'un Chérubin, une roue à côté d'un Chérubin », 10,9) ;

5. Jeffrey H. Tigay, The Evolution of the Gilgamesh Epic, Philadelphia, University of Pennsylvania Press, 1982.

6. Timothy P. Mackie, Expanding Ezekiel: The Hermeneutics of Scribal Addition in the Ancient Text Witnesses of the Book of Ezekiel, Göttingen, Vandenhoeck \& Ruprecht (Forschungen zur Religion und Literatur des Alten und Neuen Testaments FRLANT 257), 2015, p. 122-131. Voir déjà Meindert Dijkstra, «The Glosses in Ezekiel Reconsidered: Aspects of Textual Transmission in Ezekiel 10 », Ezekiel and His Book, J. Lust (ed.), Leuven University Press, 1986, p. 55-77. 
- réduction des expressions «les roues et leurs structures» / «leur aspect et leur structure » $(1,16)$ en « roues » / « aspect» respectivement $(10,9-10)$;

- plus explicatif « vers le lieu où la tête se tournait, ils allaient $(\ldots) »(10,11 \mathrm{cf} 1,17)$, mais qui ne résout pas la question de savoir de quelle tête il s'agit ;

- développement de l'anthropomorphisme des créatures comme de la présence des yeux / étincelles $(10,12$ cf 1,18). Ce développement peut être compris en regard de la rhétorique de la vision : effectivement la divinité est par excellence celle qui voit et s'oppose ainsi à l'homme et au prophète (qui a tant de peine lui à voir);

- explication et conceptualisation du système divin comme « cercle» $(10,13$ annoncé en 10,2.6) ou peut-être «tourbillon », si l'on compare les occurrences ailleurs du substantif (particulièrement en Is 5,$28 ; 17,13$; Ps 77,$19 ; 83,14$ ), ce qui permet de mieux appréhender le mouvement circulaire et la vitesse du système ;

- la transformation de la face de bovin en face de Chérubin peut se comprendre de deux manières : ou bien le Chérubin peut avoir différentes faces animales, aigle ou lion, mais pas bovine, ou bien la face d'un Chérubin est bovine, ce que semblerait confirmer la conclusion en 10,22: «Et quant à la ressemblance de leurs faces, c'étaient les faces que j'avais vues près du fleuve $\operatorname{Kevar}(\ldots) »$;

- les variations lexicales entre «s'élever» principalement en Ez 1 et «monter» en Ez 10. Mais sans doute la variante permetelle de différencier le mouvement «naturel» des créatures au mouvement «punitif» de la gloire quittant le siège de Jérusalem, comme la comparaison plus précisément entre 1,20-21 et 10,16-17 le montre.

Mais la diachronie, telle qu'elle est postulée par J. M. Hiebel ${ }^{7}$, est-elle nécessaire pour penser l'évolution de Ez 1 à 10 ? Celle-ci

7. Voir David J. Halperin, «The Exegetical Character of Ezek. X 9-17», Vetus Testamentum, 26, 1976, p. 129-141; id., The Faces of the Chariot : Early Jewish Responses to Ezekiel's Vision, Tübingen, J.C.B. Mohr (Texts and Studies in Ancient Judaism = Texte und Studien zum Antiken Judentum 16), 1988, p. 43. Contra Joachim Becker, «Ez 8-11 als einheitliche Komposition in einem pseudepigraphischen Ezechielbuch », Ezekiel and His Book, J. Lust (ed.), Leuven University Press, 1986, p. 136-150; id., Der priesterlicher Prophet. Das Buch 
ne s'inscrit-elle pas dans la logique de la structure d'ensemble du livre? Effectivement, il est clair qu'Is 6 constitue une source de la vision d'Ézéchiel. Or ce récit est formé par une première partie qui concerne la vision (Is 6,1-5) et une seconde qui concerne la mission (6,6-13). Je ne pense pas que cette bipartition soit le signe d'une trace rédactionnelle. Pourquoi le serait-elle alors dans la vision inaugurale d'Ézéchiel ? De plus, l'introduction des Chérubins en Ez 10 n'intervient qu'après la polémique contre les cultes impies et particulièrement le culte des reptiles, ce qui permet d'expliquer la non-identification des créatures vivantes avec les Séraphins, identification à laquelle on s'attend étant donné la source littéraire que constitue la vision inaugurale du livre d'Isaïe. D'autre part, comme l'avait déjà souligné D. J. Halperin, selon la narration du livre, il est logique que la vision du fleuve Kevar (Ez 1) soit clarifiée par la vision du temple (Ez 8-10): manifestement le prophète ne connaît pas les Chérubins du temple de Jérusalem avant d'y pénétrer et de les contempler en vision ${ }^{8}$ ! Les évolutions qui sont généralement analysées dans la diachronie peuvent dans ce cas se justifier sous la main d'un même scribe au vu de la logique d'ensemble. La vision est certes savamment construite mais est-elle nécessairement réécrite ? Cela ne veut pas dire que l'ensemble du rouleau est d'une même main mais que la vision continuée se prête moins facilement à un découpage tant elle est structurelle, au moins concernant la première partie jusqu'au départ de la divinité du sanctuaire?.

L'apport des sources mésopotamiennes a, par ailleurs, depuis longtemps enrichi l'interprétation de la vision d'Ézéchiel. Dans ce sens, les roues intégrées au système ne doivent pas nécessairement

Ezechiel 1-24, Stuttgart, Verlag Katholisches Bibelwerk (Stuttgarter Kleiner Kommentar : Altes Testament 12/1), 1971.

8. David J. Halperin, The Faces of the Chariot: Early Jewish Responses to Ezekiel's Vision, Tübingen, J.C.B. Mohr (Texts and Studies in Ancient Judaism $=$ Texte und Studien zum Antiken Judentum 16), 1988, p. 38.

9. La même chose ne pourrait sans doute pas être dite concernant la vision finale de la Jérusalem nouvelle en Ez 40-48. Voir en dernier lieu le point sur la question dans l'article de Dany Nocquet, "L'homme-guide d'Ézéchiel d'Ez 40-48: une manifestation anthropomorphique de Yhwh?", Entre dieux et hommes : anges, démons et autres figures intermédiaires. Actes du colloque organisé par le Collège de France, Paris, les 19 et 20 mai 2014, T. Römer et al. (eds), Fribourg - Göttingen, Academic Press - Vandenhoeck \& Ruprecht (OBO 286), 2017, p. 274-292. 
être considérées comme un ajout. Ainsi, depuis R. Dussaud, elles appartiennent à un système de représentation du divin qui intègre roues et créatures hybrides dans un système cosmologique complexe ${ }^{10}$. La vision continuée du livre d'Ézéchiel apparaît dès lors hautement construite, fruit d'une main de scribe érudit maniant sources et traditions selon un point de vue particulier qui vise à décrire un système divin, à la fois unifié mais libre, représenté mais diffus, stable mais dynamique, et de toute évidence cosmique.

Aussi, et c'est ici le dernier point mais peut-être le plus important, est-il regrettable que la partie théologique ne soulève pas les questions d'anthropomorphisme ni de représentation du divin. C'est tout de même l'une des questions les plus stimulantes auxquelles l'élite de Jérusalem a été confrontée lorsque le temple a été détruit et la présence de la divinité Yhwh remise en question.

Il semble que c'est la méthode qui doit être débattue aujourd'hui sans renoncer à la dimension historique. Peut-on faire de l'histoire dans les études bibliques sans recourir à la méthode historicocritique ? Il me semble que oui, puisque la richesse des textes mis au jour, la richesse des sites fouillés, la richesse des documentations locales et internationales, permet évidemment de resituer un texte biblique dans un contexte historique qui n'est plus son petit univers propre. C'est pour cela que la méthode historico-critique ou critique rédactionnelle, si elle ne s'ouvre pas aux sources de l'histoire aujourd'hui disponibles, me paraît non seulement datée mais également et de manière regrettable assez peu féconde.

Stephanie.anthonioz@orange.fr

10. René Dussaud, «Les visions d'Ezéchiel», Revue de l'histoire des religions, 37, 1898, p. 301-313. 